\title{
Finite Element Model for Cardiac Cell mechano-electrical stimulation
}

\author{
Pau Urdeitx Diaz ${ }^{1,2}$, Mohamed H. Doweidar ${ }^{1,2}$ * \\ ${ }^{1}$ Mechanical Engineering Department, School of Engineering and Architecture (EINA), \\ University of Zaragoza, 50018 Zaragoza, Spain; \\ ${ }^{2}$ Aragon Institute of Engineering Research (I3A), University of Zaragoza, 50018 Zaragoza, Spain \\ *Corresponding author: mohamed@unizar.es
}

\begin{abstract}
Cardiomyocyte behavior is highly dependent on the mechanical and electrical stimuli. We present a computational model, based on the FEM, to evaluate their behavior under electro-mechanical stimulation. Cell migration, adhesion, and collective behavior have been considered. Low stiffness and high alternating electric field have shown to be the best combination.
\end{abstract}

\section{Introduction}

Electrical and mechanical stimulation plays a key role in cardiac tissue development, which promotes cell differentiation, maturation, and alignment $[1,2]$. Thus, different studies have demonstrated that the contractile properties of the developed cardiac tissues depend on the adequacy of the mechanical and electrical stimuli $[3,4]$. This coupling of different stimulus makes it difficult to establish the required optimum cell culture conditions, which requires an elevated number of experimental assays. In this way, computational models can dramatically reduce the number of in-vitro experiments to establish the adequate conditions [5-7].

Here, we develop a computational model to evaluate cardiac cell response into 3D matrices in response to mechanical and electrical active stimulation. The purposed model includes cell processes such as cell differentiation, proliferation, and collective cell response, under different stimulation conditions.

\section{Material \& methods}

Cardiac cell mechanics have been defined through the evaluation of the cell membrane deformation, due to the Cell-Extracellular Matrix (ECM) interaction, using the commercial Finite Element software Abaqus, through user element subroutines (UELMAT). Mechanical (ECM stiffness) and electrical (external Electric Field) stimulation have been considered. The electric field has been varied, both in the mode of application (continuos, pulsative and alternating) and in the intensity $(50-350 \mathrm{~V} / \mathrm{m})$.

\section{Cell mechanics}

In the present cell mechanics module, cell migration and cell-cell interaction have been considered. Cell migration is considered both for individual cells and groups of cells attached with stable cell junctions. In both cases, migration is defined through the balance of the acting forces on the cell. These forces include traction forces, $\mathbf{F}_{\text {trac }}$, due to the contraction of the Actin-Myosin machinery, protrusion forces, $\mathbf{F}_{\text {prot }}$, due to the random generation of cell protrusions, electric forces, $\mathbf{F}_{\text {elec }}$, due to the electrostatic effect, and drag forces, $\mathbf{F}_{\text {drag }}$, due to the movement on a viscous ECM. Thus, the force balance is defined as $[7,8]$ :

$$
\mathbf{F}_{\text {prot }}+\mathbf{F}_{\text {trac }}+\mathbf{F}_{\text {drag }}+\mathbf{F}_{\text {elec }}=0 \text {. }
$$

Cells have been considered to be able to establish stable cell adhesions which depend on the global cell orientation, $\mathbf{G}_{p o l}$, and the cell-cell contact direction, $\mathbf{e}_{i j}$. Thus, for each pair of cells on contact, we define a stable cell adhesion by defining the projection parameter, $l_{i j}$, as:

$$
l_{i j}=\frac{\operatorname{Proj}\left(\mathbf{G}_{p o l}, \mathbf{e}_{i j}\right)}{\mathbf{G}_{p o l}},
$$

where $l_{i j}$ is 1 for aligned vectors and 0 for perpendicular vectors, and being defined stable junctions when $l_{i j}>0.5$.

\section{Cell fate}

Cell maturation, differentiation, and apoptosis have been defined based on the mechanical stimulus which the cell perceives. This stimulus has been defined through the cell membrane deformations along the cell cycle as:

$$
\gamma_{c}(t)=\frac{1}{n} \sum_{i=1}^{n} \mathbf{e}_{i}: \boldsymbol{\varepsilon}_{i}: \mathbf{e}_{i}^{T},
$$

where $\gamma_{c}(t)$ is the cell mechanical stimulus at each time step, $\boldsymbol{\varepsilon}_{i}$ is the strain tensor on each membrane node, $i$, and $\mathbf{e}_{i}$ is the direction vector from the membrane node towards the cell centroid. Thus, cell state, including cell differentiation and cell apoptosis, is defined as:

$$
\text { Cell state }=\left\{\begin{array}{cc}
m & \gamma_{\text {low }}<\gamma_{c} \leq \gamma_{\text {myo }} \\
\text { apoptosis } & \gamma_{\text {apop }}<\gamma_{c}
\end{array},\right.
$$


where $m$ corresponds to the cardiomyocyte cell phenotype, $\gamma_{\text {low }}$ and $\gamma_{\text {myo }}$ are the lower and upper bound of mechanical stimuli that trigger cardiac cell differentiation, respectively, and $\gamma_{a p o p}$ is the mechanical stimulus that triggers cell apoptosis.

\section{Conclusions}

A computational model has been developed to study the electric and mechanical stimuli on 3D matrices. This model has been applied to study the cardiac cell development on which the electric and mechanical stimuli have been demonstrated to be especially relevant. Employing the presented model, interesting conclusions have been obtained. The effect of the electric field depends on the mode of application (continuous, pulsative, or alternating electric field) and on its coupling with the mechanical stimulus. More elongated groups are obtained with higher alternating electric stimulus and low ECM stiffness.

\section{ACKNOWLEDGMENTS}

The authors acknowledge the financial support from the Spanish Ministry of Science and Innovation (PID2019106099RB-C44/AEI/10.13039/501100011033), the Government of Aragon (DGA-T24_20R), and the Biomedical Research Networking Center in Bioengineering, Biomaterials and Nanomedicine (CIBER$\mathrm{BBN})$.

\section{REFERENCES}

[1] W.L. Stoppel, et. al, Electrical and mechanical stimulation of cardiac cells and tissue constructs, Adv. Drug Deliv. Rev. 96 (2016) 135-155. https://doi.org/10.1016/j.addr.2015.07.009.
$10 \mathrm{kPa}$
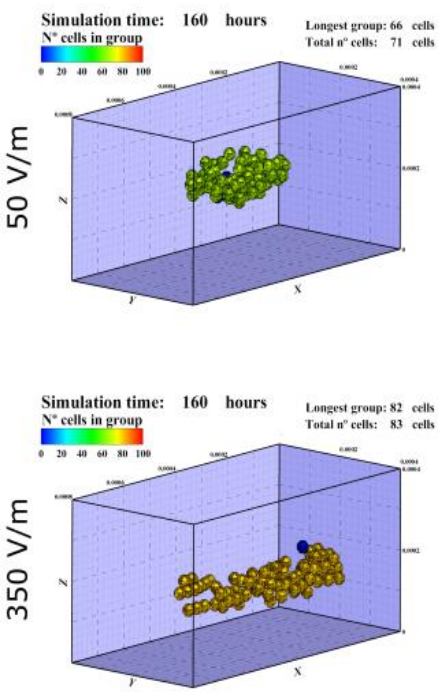

$40 \mathrm{kPa}$

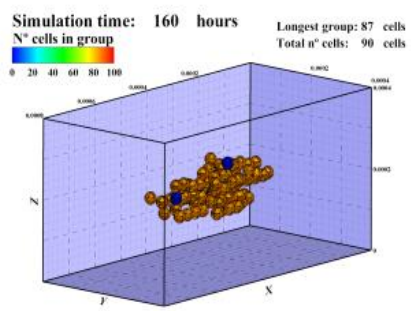

(a)

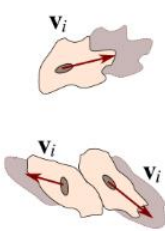

(b)

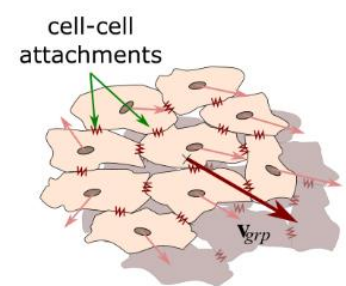

Fig. 1. Cardiac cell migration. (a) Cells migrate individually when they are not considered to be attached to other cells. (b) Collective cell migration is considered for groups of cells with strong cell adhesions.
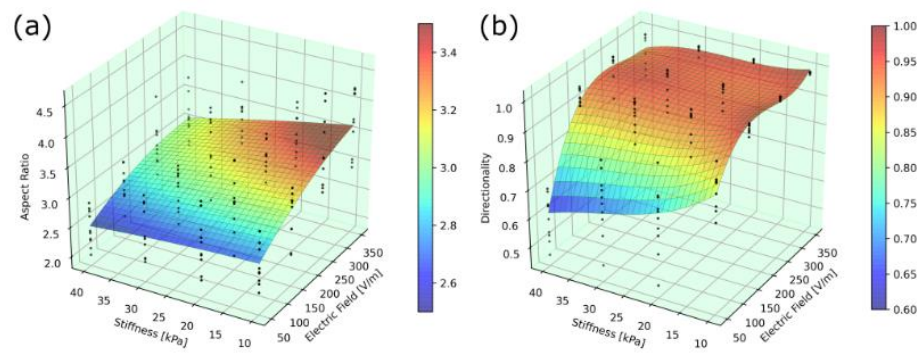

Fig. 3. Cardiac cell groups Aspect Ratio (a) and cells Directionality (b) for different ECM stiffnesses and electric field intensities. A higher effect of the electric field is observed with low ECM stiffness.
Fig. 2. Cell response to the coupling effect of the mechanical and electrical stimuli. Elongated groups are formed on the softer ECM and with maximum alternating electric stimulation.
[2] M.N. Hirt, et. al, Functional improvement and maturation of rat and human engineered heart tissue by (2014) $151-161$ https://doi.org/10.1016/j.yjmcc.2014.05.009. advanced functional maturation of human ESC-derived cardiomyocytes, Biomaterials. 34 (2013) 5813-5820. https://doi.org/10.1016/j.biomaterials.2013.04.026. maturation and scale-up of human pluripotent stem cellengineered heart tissues, Nat. Commun. (2017) 1825. https://doi.org/10.1038/s41467-017F. Galbusera, et. al, An in silico bioreactor for simulating laboratory experiments in tissue engineering, Biomed. Microdevices. 10 (2008) 547-554. https://doi.org/10.1007/s10544-008-9164-9.

Bioreactors in 3D Cell Culture and Tissue Manufacturing, Adv. Mater. 21 (2009) 3352-3367. https://doi.org/10.1002/adma.200802748. Enhance Cardiac Tissue Regeneration, Mathematics. 8 (2020) 1875. https://doi.org/10.3390/math8111875. regeneration: a 3D in-silico model, Comput. Mech. 66 (2020) 1003-1023. https://doi.org/10.1007/s00466020-01882-6.

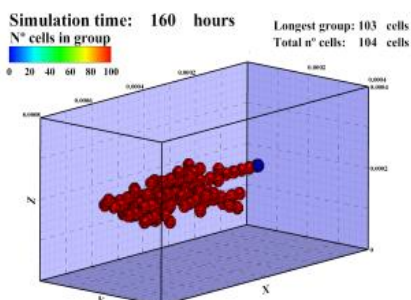

Revista “Jornada de Jóvenes Investigadores del I3A”, vol. 8 (Actas de la IX Jornada de Jóvenes Investigadores del I3A -11 de diciembre de 2020). ISSN 2341-4790. 